\title{
Application of multi-sensor Information Fusion Fire Early Warning System in Intelligent Fire Protection Field
}

\author{
Dayan Sun ${ }^{1}$, Shishuai Zhang ${ }^{2}$, Guang Chen², Wei Chang ${ }^{2}$, Mingmin Wang ${ }^{3}$, \\ Pengyu Guo ${ }^{3}$
}

${ }^{1}$ State grid corporation of China, China

${ }^{2}$ Nari group corporation, China

${ }^{3}$ State Grid Jiangsu Electric Power CO. LTD, China

\begin{abstract}
With the development of MEMS technology, the measurement and determination of many sensitive parameters have been realized, and the interconnection between intelligent sensor network and everything has become possible in hardware. Various sensor integrated information sensing technologies have also made great progress based on this. So, we can construct an intelligent network system based on multi-sensor system and apply it to various fields, thus making many kinds of intelligent system applications. This paper discusses and studies the application of multi-sensor information fusion fire warning system in intelligent firefighting system.
\end{abstract}

Keywords: sensors, fire early warning, intelligent fire fightingem.

\section{Brief Introduction}

Intelligent fire fighting system is a security system based on sensor technology and intelligent information processing technology. It is equipped with telephone, automatic fire extinguishing control cabinet and fire alarm broadcasting system on the alarm of fire fighting center. Once a fire occurs, the intelligent fire alarm system can immediately send an alarm signal on the fire alarm in the region, and at the same time, the alarm signal is sent out on the alarm equipment of the fire center, and the location or area code of the fire is displayed. When the manager receives the alarm, he immediately starts the fire broadcast, organizes the safety evacuation of the personnel, and starts the fire power. Ladder; alarm linkage signal to drive the automatic fire extinguishing control cabinet to work, close the fire door to close the fire area, and spray water or fire extinguishing agent in the fire area; start fire pump and automatic smoke exhaust device. In the early prediction of fire, the situation of fire and the location of fire, the trend of spread can only be distinguished based on the comprehensive signals of multi-sensor system, and can be displayed in the building and other threedimensional structures, and the distribution of nearby personnel can be based on such information to arrange evacuation and rescue as early as possible to reduce search. Time to save personnel. In the mid-term of a fire, the trend and direction of possible spread can be analyzed based on the situation of the fire, and the corresponding response can be done early on this basis. In the later stage of the fire, we can use the data on the server to analyze the large data, so as to warn the fire prevention. In this paper, based on multi-sensor information fusion technology for fire identification and early warning, a multi-sensor fusion system for fire warning system is constructed.

\section{Construction of Distributed Fire Sensor System}

Different sensor systems are constructed according to different fire characteristics: including but not limited to smoke sensors, carbon dioxide sensors, temperature sensors, infrared sensors, and the corresponding wireless forwarding module, power management module, etc. 


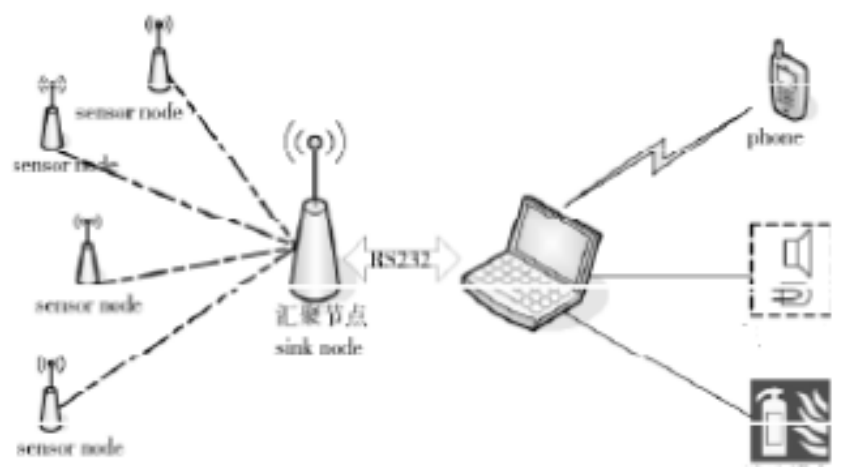

Fig 1. distributed sensor network

Through the distributed deployment of multi-sensor nodes, the indoor multi-point temperature and humidity, smoke and gas concentration and other information are detected respectively, so as to accurately grasp the characteristics of the pre-fire, the overall structure is shown in Figure 1. Considering the characteristics of indoor environment, the system adopts star network topology, and defines two kinds of sensors, i.e. convergence node and sensor node. Star network adopts point-topoint communication mode, each sensor node can only communicate with the only sink node in the network, and any two sensor nodes can not communicate directly, so the data transmission is safe. But at the same time, the security of the sink node should be guaranteed, otherwise the sink node will disrupt or even destroy part of the sensor network, and make the information invalid.

Each sensor will collect data collected by MCU for data processing and give out the result of the decision. The wireless transceiver data from the RF unit is transmitted through the network to the $2.4 \mathrm{GHz}$ wireless channel and converged to the sink node. The sink node transmits the data to the master server through the serial port circuit, and judges the situation by the master server's data fusion algorithm and data processing, finally decides whether to issue the warning.

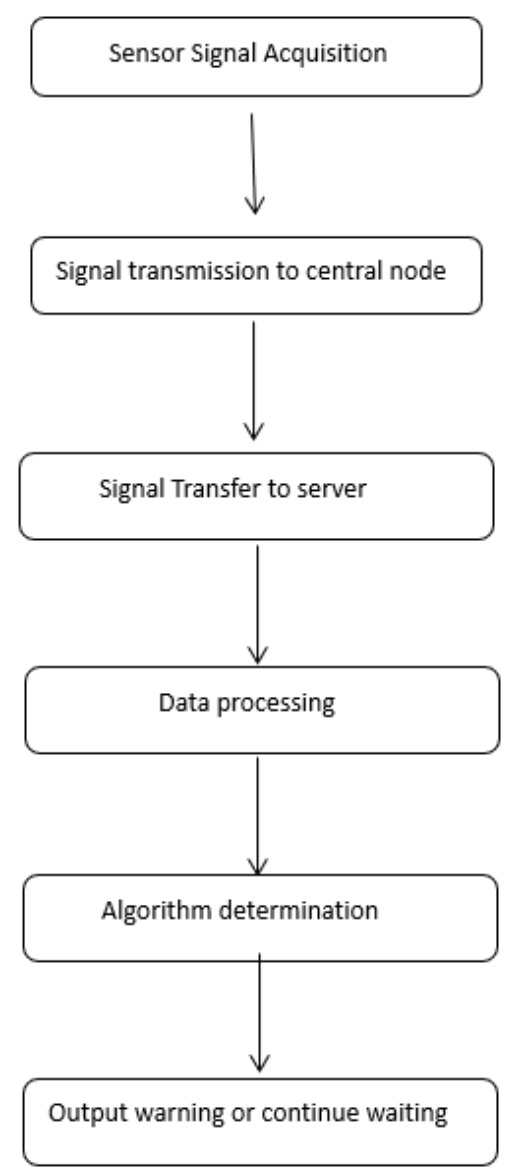

Fig 2. work mode flow of multi-sensor information system 


\section{Fire Warning Identification Algorithm}

There are many kinds of algorithms for judging whether a fire happens or not. In this paper, a combination of weighted synthesis algorithm and analytic hierarchy process is used to judge whether a fire happens or not. The specific algorithm is as follows:

The degree and importance of sensors are divided into three levels, marked A, B and C. The degree of importance descends in turn, and the weight of the first level is the highest and decreases in turn. As data source processing.

The basic principle of multi-sensor data fusion technology is just like that of human brain integrated processing information. By making full use of multi-sensor resources and reasonably controlling and using multi-sensor and its observation information, the redundant or complementary information of multi-sensor in space or time is combined according to some criterion to obtain the measured pair. An interpretation or description of the consistency of an image. Specifically, the principle of multi-sensor data fusion is as follows:

(1) $\mathrm{N}$ different types of sensors (active or passive) collect data for observation targets.

(2) Transform the output data of the sensor (discrete or continuous time function data, output vector, imaging data or a direct attribute description) to extract the feature vector Yi representing the observed data.

(3) The feature vector I is processed by pattern recognition (e.g. clustering algorithm, adaptive neural network or other statistical pattern recognition method which can transform the feature vector I into target attribute decision) to complete the description of the target by each sensor.

(4) grouping the description data of each sensor on the same target according to the target, that is, association.

(5) The fusion algorithm is used to synthesize the sensor data of each target and get the consistency interpretation and description of the target.

Finally, Bayesian algorithm is used for pattern recognition and judgment. Bayesian estimation provides a means for data fusion and is a common method for fusion of multi-sensor high-level information in static environment. It makes sensor information be combined according to the principle of probability. Measurement uncertainty is expressed by conditional probability. When the observation coordinates of sensor group are consistent, sensor data can be fused directly. But in most cases, sensor data should be fused indirectly by Bayesian estimation. Dobby estimation takes each sensor as a Bayesian estimation and combines the probability distribution of each individual object into a joint posterior probability distribution function. By using the likelihood function of the joint distribution function as the minimum, the final fusion value of the multi-sensor information is provided, which fuses the information and the environment first. The model provides a characteristic description of the whole environment. Based on the results of several significant models, the results of data are calibrated and analyzed.

Finally, we use Calman filter to conduct fire warning model learning and analysis. Kalman filter is usually used to estimate and correct the information. Its effect on multi-sensor information fusion is not only a simple and specific algorithm, but also a very useful system processing scheme. In fact, it is similar to many systems in processing information data. It uses the mathematical iterative recursive computation method to provide an effective optimal estimation in statistical sense for data fusion. However, it requires very little storage space and computation, and is suitable for the environment with limited data processing space and speed. KF is divided into two kinds: decentralized Calman filtering (DKF) and extended Calman filtering (EKF). DKF can make data fusion completely decentralized, and EKF can effectively overcome the impact of data processing errors and instability on the process of information fusion.

\section{Summary}

The Bayesian mode multi-sensor network information fusion model based on Kalman filter is useful for fire prevention, fire control, rescue and evacuation, but the key data nodes of the distributed 
system need more protection. At the same time, the wake-up signal should be selected according to historical data.

\section{References}

[1]. Wang Jun, Su Jianbo, Xi Yu Gen. summary of multi-sensor fusion [J]. data acquisition and processing, 2004, 19 (1): 72-77.

[2]. Li Shengyi, Wu Xuezhong, Fan Dapeng. Multisensor Fusion Theory and Its Application in Intelligent Manufacturing System [M]. National University of Defense Science and Technology Press, 1998.

[3]. Zhang Zhenhao, Sun Wei, Zhao Chunyu.Multi-sensor fused UAV dynamic autonomous landing system[J].Optical Precision Engineering, 2017, 25 (10s): 151-159.

[4]. Zhang Jing. Application of multi-sensor information fusion in fire detection [D]. Guangdong University of Technology, 2003.

[5]. Liu Xiaojun, Bao Hong.Realization of fire alarm system based on ARM9 multi-sensor data fusion[J].Industrial control computer, 2009,22(3): 52-53.

[6]. Li Zhengzhou, Fang Chaoyang, Gu Yuanshan, et al. Fire detection system based on wireless multi-sensor information fusion [J]. Data acquisition and processing, 2014, 29 (5): 694-698.

[7]. Zhang Pin, Dong Weihao, Gao Dadong. An optimized Bayesian estimation multisensor data fusion method [J].Acta Sensing Technology, 2014 (5): 643-648.

[8]. Chen Xiaolong, Wang Jiali, Sun Lu, et al. A multi-sensor data fusion method based on Bayesian estimation [C]//TTC, metrology, instrumentation and instrumentation annual meeting. 2009.

[9]. Chen Zhigang, Li Xian, Xu Shiyou, et al. Airborne Multisensor Performance Assessment of Bayesian Network Fusion Model [J]. High-tech Communications, 2013, 23 (10): 993-1000.

[10]. Cui Pingyuan,Huang Xiaorui.Multi-sensor information fusion algorithm based on joint Kalman filter and its application[J].Acta Motor and Control,2001,5(3): 204-207.

[11]. Sheng Sanyuan,Wang Jianhua.Application of Joint Kalman Filter in Multi-sensor Information Fusion[J].Radar and Countermeasure,2002(1): 27-33.

[12]. Li Haiyan, Li Weijia, Huang Yunbao.Multisensor Data Fusion Based on Kalman Filter[J].Journal of Wuhan University (Engineering Edition), 2011, 44 (4): 521-525. 